Check for updates

Cite this: RSC Adv., 2019, 9, 6241

Received 10th January 2019

Accepted 14th February 2019

DOI: 10.1039/c9ra00214f

rsc.li/rsc-advances

\section{Antibacterial nanoparticles: enhanced antibacterial efficiency of coral-like crystalline rhodium nanoplates $\uparrow$}

\author{
Woojun Shin, $\ddagger^{a}$ Hyuk Seung Han, $\star^{\mathrm{b}}$ Nghia T. K. Le, ${ }^{\mathrm{b}}$ Kyungtae Kang ${ }^{\star b}$ \\ and Hongje Jang (iD *a
}

\begin{abstract}
This paper deals with the newly found antibacterial efficiency of coral-like crystalline Rh nanoplates. Rh nanoplates with rough surface morphology synthesized by inverse-directional galvanic replacement exhibited highly enhanced antibacterial efficiency compared to $\mathrm{Rh}^{3+}$ ion and $\mathrm{Rh}$ nanospheres. The observed antibacterial efficiency was comparable to Ag nanoplates, a well-known anticancer nanoagent. Results clearly demonstrate that the composition and morphology of a nanostructure play significant roles in antibacterial effects.
\end{abstract}

Bacteria live everywhere, covering most of where we live. Although some bacteria such as lactobacilli are beneficial to humans, the risks of chronic infection and sepsis caused by a bacterial infection also exist. ${ }^{1}$ Although the use of antibiotics is widely accepted as a general countermeasure against infections, drug overuse has brought about the emergence of super bacteria, multidrug-resistant bacterial strains that can be considered as a major crisis. ${ }^{2}$ As a new direction in developing antibiotics, approaches using antibacterial nanoparticles have attracted much attention. ${ }^{3}$ Typical examples include Grampositive antibacterial metal oxides such as zinc oxide ( $\mathrm{ZnO})$ and titanium dioxide $\left(\mathrm{TiO}_{2}\right),{ }^{4,5}$ and Gram-negative antibacterial metal nanoparticles such as silver (Ag) nanoparticles. ${ }^{6}$ Antibacterial effects of nanoparticles are known to rely on bacterial cell membrane disruption, ${ }^{7}$ reactive oxygen species generation, ${ }^{8}$ and intracellular antibacterial effects. ${ }^{9}$ For nanoparticles have such abilities, small dimensions and positive surface charge tend to be more favorable, but the exact features of inorganic nanostructures responsible for antibacterial effect remain to be elucidated. Therefore, the discovery of antibacterial nanoparticles based on new elements, morphology, composition, and physicochemical properties still merits efforts.

Synthesis via surface control or post-synthetic treatments are generally utilized to manufacture fascinating nanostructures with various shapes and elemental compositions..$^{10}$ Among them, galvanic replacement based on spontaneous redox

\footnotetext{
${ }^{a}$ Department of Chemistry, Kwangwoon University, 20, Gwangwoon-ro, Nowon-gu, Seoul 01897, Republic of Korea. E-mail: hjang@kw.ac.kr

${ }^{b}$ Department of Applied Chemistry, Kyung Hee University, Yongin, Gyeonggi 446-701, Republic of Korea. E-mail: kkang@khu.ac.kr

$\dagger$ Electronic supplementary information (ESI) available. See DOI: 10.1039/c9ra00214f

$\$$ The authors are equally contributed.
}

reactions between oxidation-prone nanotemplates and reduction-prone replacing metal cations is generally used, since it is environment-friendly and convenient to perform. ${ }^{11}$ In typically such galvanic replacement setups, $\mathrm{Ag}$ or copper $(\mathrm{Cu})$ for nanotemplates, and gold ( $\mathrm{Au})$, platinum $(\mathrm{Pt})$, and palladium (Pd) for replacing cations, many of which are closely related to antibacterial properties. $^{12}$ Therefore, we expected galvanic replacement can be a promising way to develop new antibacterial nanoparticles.

In this study, we identified the antibacterial property of coral-like crystalline rhodium nanoplates (RhNPs), which recently emerged for its new possibilities in biomedical applications. ${ }^{13}$ To verify the enhanced antibacterial activity of RhNPs based on the morphology and composition, a comparative experiment was performed using $\mathrm{Ag}$ nanoplates (AgNPs), conventionally small $\mathrm{Rh}$ nanospheres (RhNSs; $5 \mathrm{~nm}$ diameter), and $\mathrm{Rh}^{3+}$ ions (Fig. 1). ${ }^{14}$ Quantitative analysis showed that RhNPs exhibited a slightly improved effect when compared to AgNPs (typically used as an antibacterial nanomaterial), ${ }^{15}$ and free $\mathrm{Rh}$ ions and $\mathrm{Rh}$ nanospheres were not effective. The results not only demonstrate a new possibility of using metal elements beyond $\mathrm{Ag}$ for antibacterial nanostructures, but also show that structural properties play an important role.

RhNP preparation was accomplished using a two-step procedure: seed-mediated synthesis of sacrificial Ag nanoplate templates, followed by inverse-directional galvanic replacement with $\mathrm{Rh}^{3+}$. The high-temperature $\left(190{ }^{\circ} \mathrm{C}\right)$ reaction along with the reducing agent and ethylene glycol (EG) co-solvent condition, which caused galvanic replacement depending on the shape of the AgNPs. A subsequent outward surface growth resulted in the formation of coral-like RhNPs. Similarly, control RhNS synthesis was accomplished via reductant-based synthesis under the same conditions without $\mathrm{Ag}$ 


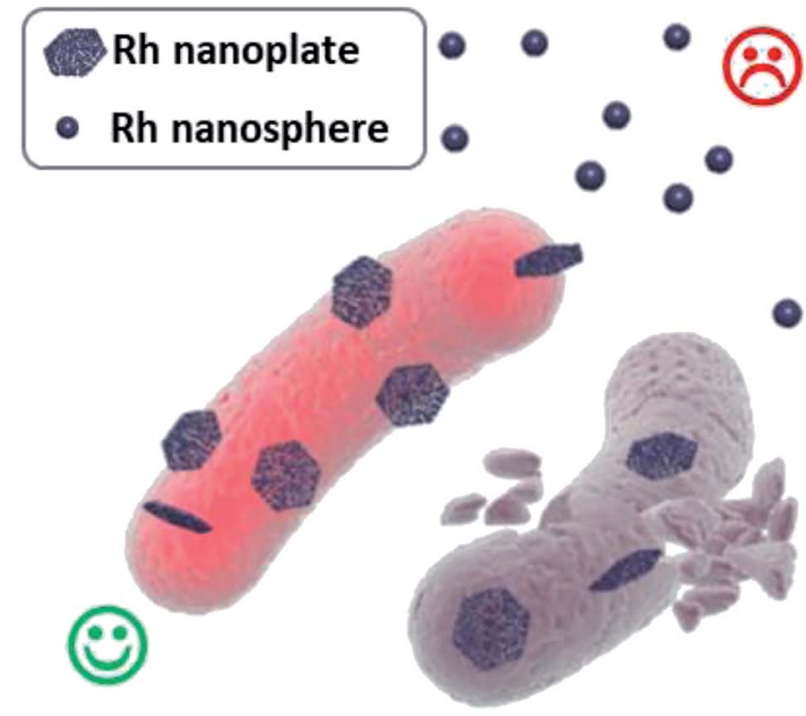

Fig. 1 Schematic illustration of the antibacterial effect of Rh nanoparticles in spherical and coral-like plate morphologies.

nanotemplates. According to the transmission electron microscopy (TEM) and scanning electron microscopy (SEM) images, approximately $120 \mathrm{~nm}$ long (transverse length) RhNPs with rough surface morphologies were produced successfully (Fig. 2a and $\mathrm{S} 1 \dagger$ ). The formation of RhNPs was accomplished by rapid galvanic replacement of AgNPs template with $\mathrm{Rh}^{3+}$ ions by spontaneous redox reaction at high temperature. The manufactured nanostructures showed a nanoplatelet structure caused by rapid galvanic replacement of template and outwardgrown coral-like morphology by subsequent secondary growth.
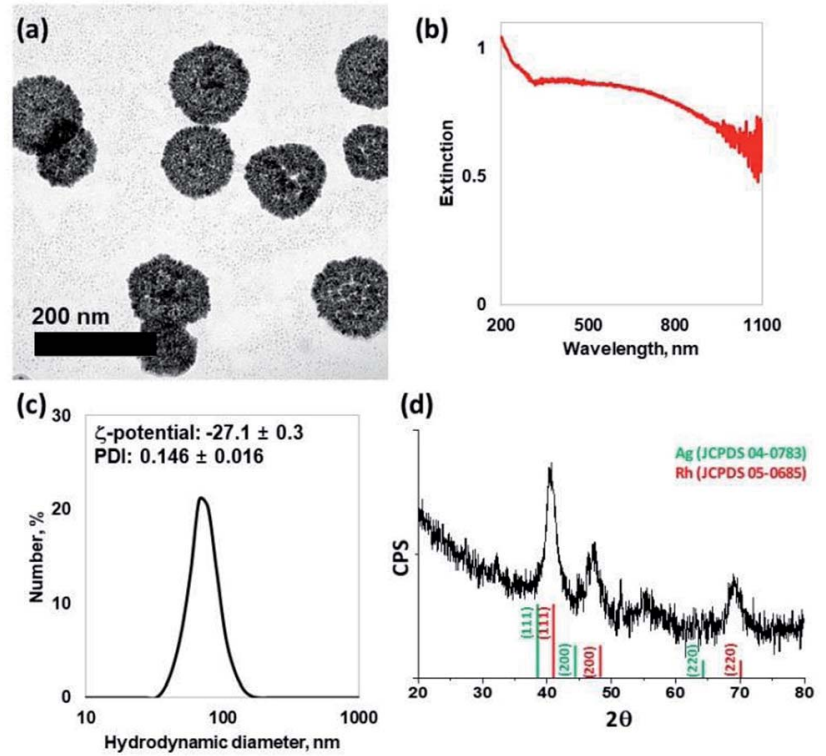

Fig. 2 Characterization of prepared RhNPs. (a) TEM, (b) UV-Vis spectrometer, (c) DLS and zeta-potential, and (d) XRD pattern exhibited the successful synthesis of crystalline monodisperse RhNPs. The scale bar is $200 \mathrm{~nm}$.
Coral-like nanoplates with high surface-to-volume ratio and many surface irregularities have a high potential for intimate interactions with bacterial or mammalian cell membranes.

The extinction spectrum of RhNP identified through UVvisible-near infrared spectroscopy showed a weak UV-localized plasmon resonance peak at $273 \mathrm{~nm}$ and uniformly high optical density over the whole wavelength region with weak and broad bands at around 320-1100 nm, which probably originated from the coral-like two-dimensional interconnected complex structure (Fig. 2b). The hydrodynamic diameter of RhNP was observed to be $68.1 \mathrm{~nm}$ for maximum number distribution, which is smaller than that measured using the TEM images, because calculations carried out using the StocksEinstein equation assume a spherical shape. Although the polydispersity index (PDI) was excellent $(0.146 \pm 0.016)$, it did not satisfy the recommended parameters for antibacterial nanoparticle efficacy due to the highly negative surface charge

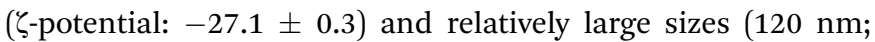
Fig. 2c).

High-resolution (HR)-TEM observations and fast Fourier transforms of the images provided highly crystalline facecentered cubic (fcc) structures (Fig. S2 $\dagger$ ). Moreover, a slight shift from the X-ray diffraction pattern was observed possibly due to the partially-presenting $\mathrm{Rh}-\mathrm{Ag}$ alloy composition (Fig. 2d).

To investigate the antibacterial effect of RhNP further, three additional control materials were prepared and their antibacterial properties against Escherichia coli were tested. AgNPs, a well-known antibacterial material and a template for galvanic replacement, were prepared using the same particle count as RhNPs to identify constituent element-based differences. In addition, $\mathrm{Rh}^{3+}$ ions and $\mathrm{RhNS}$ were prepared with the same $\mathrm{Rh}$ content via inductively-coupled plasma mass spectroscopy. All nanoparticles were prepared at a similar degree of surface negative charge, which excluded the antibacterial effect from the charge property (Fig. S3 and S4 $\dagger$ ).

Compared to untreated control (negative), RhNPs showed remarkable inhibition of $E$. coli growth; the inhibition was even slightly better than AgNPs (positive) when treated at the same concentration. Contrastingly, free $\mathrm{Rh}^{3+}$ ions and RhNSs did not show noticeable effects even though they had the same Rh contents. From these results, both the constituent element and morphological characteristics were confirmed to be crucial factors (Fig. 3a). Similar results were also obtained for the dosedependent antibacterial effects of RhNPs (Fig. 3b).

SEM images of RhNP-treated $E$. coli provided more detailed information regarding the observed antibacterial effects. Surface adsorption of a large number of nanoplates was observed in $E$. coli treated with RhNPs when compared to control (Fig. 4a and b). Magnified SEM images (Fig. 4c and d) show that RhNPs were adsorbed in a form of clusters on the surfaces of $E$. coli. The results also indicated that RhNPs bigger than a certain size were required for interaction with $E$. coli surfaces, and that relatively small RhNPs were ineffective due to difficulties in accessing and interacting with the $E$. coli surface. Negatively-charged particles may seem hard to adhere to cells due to the negatively-charged cell membrane, so the adhesion 

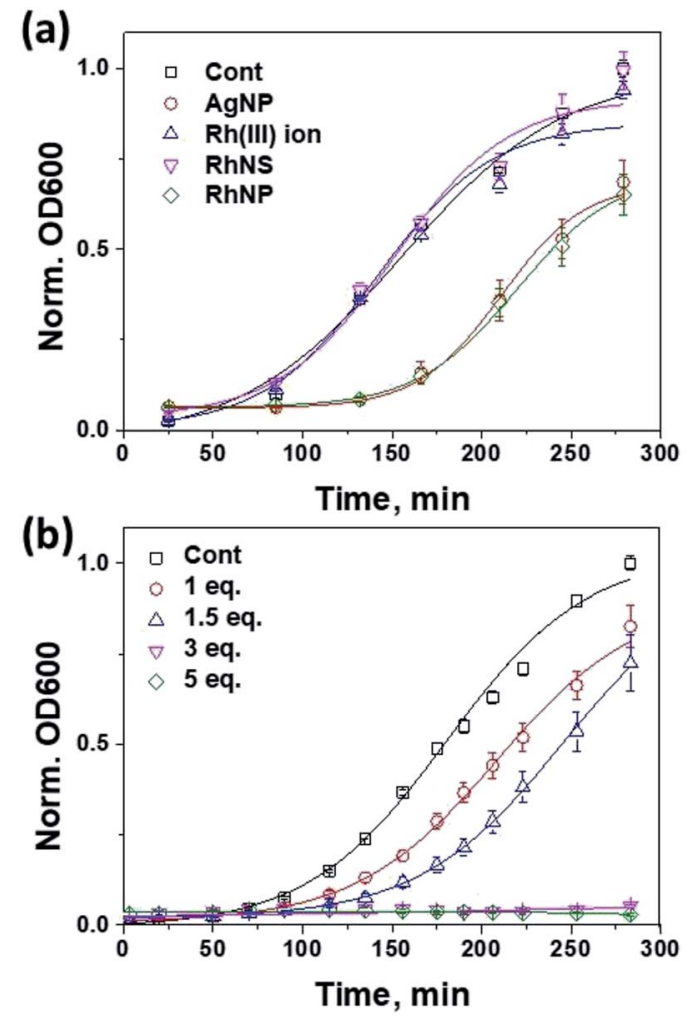

Fig. 3 Growth curve of $E$. coli as an optical density $\left(O D_{600}\right)$ plot. Comparison against (a) nanomaterials and (b) RhNP concentrations exhibited significant antibacterial efficiency.

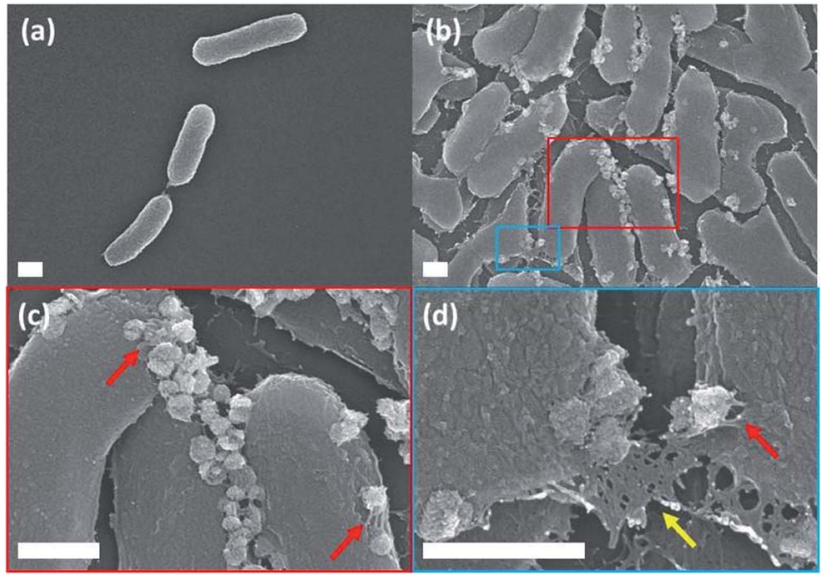

Fig. 4 SEM images of (a) control- and (b) RhNP-treated E. coli clearly exhibiting surface adsorption of the nanoplates. The red (c) and blue (d) boxes are enlarged images of the red-and blue-boxed regions in (b). Red arrows indicate the surface interaction between $E$. coli and RhNPs, and the yellow arrow indicates a ruptured $E$. coli cell. The scale bar is $500 \mathrm{~nm}$.

presumably occurred between RhNPs and protein corona clusters. Such adsorption of RhNPs resulted in cell membrane weakening (Fig. 4c and d; red arrows) or disruption (Fig. 4d; yellow arrow), which account for the observed antibacterial property.

\section{Conclusion}

In this study, we reported the antibacterial effect of RhNPs that varies depending on their chemical composition and morphology. Recent studies based on RhNPs with contact lengths of $100 \mathrm{~nm}$ or more showed interesting results. Notably, previous studies reported difficulties in synthesizing $\mathrm{Rh}$ nanoparticles above $5 \mathrm{~nm}$. Although the actual antibacterial effects and partial mechanisms of RhNP were identified in the present study, specific factors for the RhNP antibacterial properties were not elucidated. However, it is important to continue exploring the biological applications of $\mathrm{Rh}$, because the antibacterial effects of nanomaterials still remain unclear.

\section{Conflicts of interest}

There are no conflicts to declare.

\section{Acknowledgements}

This work was supported by the Grant No. (NRF2016R1C1B1008090) through the National Research Foundation of Korea (NRF) and a grant from Kyung Hee University in 2016 (KHU-20160610).

\section{Notes and references}

1 (a) S. S. Grant and D. T. Hung, Virulence, 2013, 4, 273; (b) S. Li, S. Dong, W. Xu, S. Tu, L. Yan, C. Zhao, J. Ding and X. Chen, Adv. Sci., 2018, 5, 1700527; (c) R. A. Fisher, B. Gollan and S. Helaine, Nat. Rev. Microbiol., 2017, 15, 453; (d) D. J. Stearns-Kurosawa, M. F. Osuchowski, C. Valentine, S. Kurosawa and D. G. Remick, Annu. Rev. Phytopathol., 2011, 6, 19.

2 (a) P. R. Hsueh, J. Formosan Med. Assoc., 2010, 109, 685; (b) A. Salman, U. Sharaha, E. Rodriguez-Diaz, E. Shufan, K. Riesenberg, I. J. Bigio and M. Huleihel, Analyst, 2017, 142, 2136; $(c)$ K. Timraz, Y. Xiong, H. A. Qarni and P. Y. Hong, Environ. Sci.: Water Res. Technol., 2017, 3, 293.

3 (a) L. Wang, C. Hu and L. Shao, Int. J. Nanomed., 2017, 12, 1227; (b) R. Singh, M. S. Smitha and S. P. Singh, J. Nanosci. Nanotechnol., 2014, 14, 4745; (c) E. D. Cavassin, L. F. de Figueiredo, J. P. Otoch, M. M. Seckler, R. A. de Oliveira, F. F. Franco, V. S. Marangoni, V. Zucolotto, A. S. S. Levin and S. F. Costa, J. Nanobiotechnol., 2015, 13, 64; (d) L. Wang, C. Hu and L. Shao, Int. J. Nanomed., 2017, $12,1227$. 4 (a) A. Sirelkhatim, S. Mahmud, A. Seeni, N. H. M. Kaus, L. C. Ann, S. K. M. Bakhori, H. Hasan and D. Mohamad, Nano-Micro Lett., 2015, 7, 219; (b) J. Lin, J. Ding, Y. Dai, X. Wang, J. Wei and Y. Chen, Mater. Sci. Eng., C, 2017, 81, 321.

5 A. Kubacka, M. S. Diez, D. Rojo, R. Bargiela, S. Ciordia, I. Zapico, J. P. Albar, C. Barbas, V. A. P. Martins dos Santos, M. Fernández-Garcia and M. Ferrer, Sci. Rep., 2014, 4, 4134.

6 (a) J. S. Kim, E. Kuk, K. N. Yu, J. H. Kim, S. J. Park, H. J. Lee, S. H. Kim, Y. K. Park, Y. H. Park, C. Y. Hwang, Y. K. Kim, 
Y. S. Lee, D. H. Jeong and M. H. Cho, Nanomedicine, 2007, 3, 95; (b) B. L. Ouay and F. Stellacci, Nano Today, 2015, 10, 339.

7 (a) D. Depan and R. D. Misra, Mater. Sci. Eng., C, 2014, 34, 221; (b) X. Xu, D. Chen, Z. Yi, M. Jiang, Z. Zhou, X. Fan, Y. Wang and D. Hui, Langmuir, 2013, 29, 5573; (c) Y. N. Slavin, J. Asnis, U. O. Häfeli and H. Bach, J. Nanobiotechnol., 2017, 15, 65.

8 (a) M. Hyldgaard, T. Mygind, B. S. Vad, M. Stenvang, D. E. Otzen and R. L. Meyer, Appl. Environ. Microbiol., 2014, 80, 7758; (b) H. A. Hoster, I. B. Ditta, S. Varghese and A. Steele, Appl. Microbiol. Biotechnol., 2011, 90, 1847; (c) M. Y. Memar, R. Ghotaslou, M. Samiei and K. Adibkia, Infect. Drug Resist., 2018, 11, 567.

9 (a) Y. Su, X. Zheng, Y. Chen, M. Li and K. Liu, Sci. Rep., 2015, 5, 15824; (b) M. Yamanaka, K. Hara and J. Kudo, Appl. Environ. Microbiol., 2005, 71, 7589; (c) H. Mu, J. Tang, Q. Liu, C. Sun, T. Wang and J. Duan, Sci. Rep., 2016, 6, 18877.

10 (a) K. Chatterjee, S. Sarkar, K. J. Rao and S. Paria, Adv. Colloid Interface Sci., 2014, 209, 8; (b) M. J. Mulvihill, X. Y. Ling, J. Henzie and P. Yang, J. Am. Chem. Soc., 2010, 132, 268.
11 (a) A. G. M. da Silva, T. S. Rodrigues, S. J. Haigh and P. H. C. Camargo, Chem. Commun., 2017, 53, 7135; (b) H. Jang, Y. K. Kim and D. H. Min, Chem. Commun., 2017, 53, 1385.

12 (a) S. Kang, K. Kang, H. Huh, H. Kim, S. J. Chang, T. J. Park, K. S. Chang, D. H. Min and H. Jang, ACS Appl. Mater. Interfaces, 2017, 9, 35268; (b) S. Kang, W. Shin, K. Kang, M. H. Choi, Y. J. Kim, Y. K. Kim, D. H. Min and H. Jang, ACS Appl. Mater. Interfaces, 2018, 10, 13819.

13 S. Kang, W. Shin, M. H. Choi, M. Ahn, Y. K. Kim, S. Kim, D. H. Min and H. Jang, ACS Nano, 2018, 12, 6997.

14 (a) V. Cimpeanu, M. Kočevar, V. I. Parvulescu and W. Leitner, Angew. Chem., Int. Ed., 2009, 48, 1085; (b) R. R. Dykeman, N. Yan, R. Scopelliti and P. J. Dyson, Inorg. Chem., 2011, 50, 717; (c) Y. Yuan, N. Yan and P. J. Dyson, ACS Catal., 2012, 2, 1057.

15 A. D'Agostino, A. Taglietti, R. Desando, M. Bini, M. Patrini, G. Dacarro, L. Cucca, P. Pallavicini and P. Grisoli, Nanomaterials, 2017, 7, 7. 